\title{
IMPACT OF MULTIPATH ANGULAR Distribution on Performance of MIMO Systems
}

\author{
Leo Chan ${ }^{1,2}$, Sergey Loyka ${ }^{2}$ \\ ${ }^{1}$ Nortel Networks, 3500 Carling Avenue, Ottawa, Ontario, Canada, K2H 8E9 \\ ${ }^{2}$ School of Information Technology and Engineering (SITE) \\ University of Ottawa, 161 Louis Pasteur, Ottawa, Ontario, Canada, K1N 6N5
}

\begin{abstract}
The objective of this paper is to analyze the impact of multipath angular distribution on the channel correlation and performance of MIMO systems. A full breadth of AOA models is used in this study, and they cover most scattering scenarios of the physical propagation channel. Closed-form expressions for parameters and corresponding correlation of these AOA models are derived. A simulation model was developed and extensive Monte Carlo simulations were performed to obtain the MIMO channel capacity, correlation, and diversity gain for various scenarios. Two major results are (i) the distributions shape has minor impact of the correlation, capacity and diversity gain; the major impact is due to the angular spread; (ii) the continuous and impulsive distribution have dramatically different impacts on the correlation, capacity and diversity gain.
\end{abstract}

Keywords: wireless communications, MIMO, correlation.

\section{INTRODUCTION}

MIMO (multiple-input-multiple-output) systems have emerged as one of the major technological breakthrough in the field of wireless communications in recent years. The transformation of a multipath channel into parallel subchannels offers tremendous improvement in data rate. By properly exploiting these sub-channels, MIMO systems offer a theoretical upper limit on capacity that increases almost linearly with the number of antenna elements in rich multipath environments. This has opened up many opportunities and spawned numerous research activities.

Much of the recent activities on MIMO systems have focused on the development of channel models, derivations of the MIMO channel capacity, and identification of relationships between system parameters and achievable channel capacity. The objective of this paper is to produce a comprehensive study on the impact of multipath angular distribution on the performance of MIMO systems. As part of this study, different distributions are used to emulate the AOA of multipath signals. These distributions are carefully chosen to represent a full breadth of possible angle of arrival (AOA) distributions that can occur in real-life environment. The parameters that are studied include correlation, channel capacity, outage probability, and diversity gain.

\section{SYSTEM MODEL}

Without loss of generality, we consider a MIMO system equipped with $n \mathrm{Tx}$ and $n \mathrm{Rx}$ antennas. The wireless channel is assumed to have a scattering environment where multipath components arrive at the receiver from different directions in a 2-D (horizontal) plane; the direction is characterized by the azimuth (i.e. AOA). The received signal at the $i^{\text {th }}$ receive antenna element from the $j^{\text {th }}$ transmit antenna element is [1]:

$$
h_{i j}=\sum_{k=1}^{N} a_{k} e^{j\left(\varphi_{k}-\beta_{i k}\right)}
$$

where $N$ is the number of multipath components for each transmitted signal, $a_{k}$ and $\varphi_{k}$ are the amplitude and phase of the $\mathrm{k}^{\text {th }}$ multipath component, and $\beta_{i k}$ is the phase shift of the $\mathrm{k}^{\text {th }}$ multipath component in the $\mathrm{i}^{\text {th }} \mathrm{Rx}$ antenna with respect to the first one, $\beta_{i k}=(2 \pi / \lambda) d(i-1) \cos \left(\theta_{k}\right)$, where $\lambda$ is the wavelength, $d$ is the $\mathrm{Rx}$ antenna spacing, and $\theta_{k}$ is the AOA of $\mathrm{k}$-th multipath. Clearly, we consider quasi-static and frequency-flat channel. Only the Rx end correlation is accounted for (assuming zero correlation at the $\mathrm{Tx}$ end). If required, the $\mathrm{Tx}$ end correlation can be modeled in the same way. The instantaneous correlation matrix is defined as

$$
\mathbf{R}=\mathbf{H H}^{+}
$$

where $\mathbf{H}$ is the channel matrix with the entries $\left\{h_{i j}\right\}$, and + denotes Hermitian conjugate. The correlation matrix is normalized as follows: $\operatorname{tr}[\mathbf{R}]=n$.

\section{A. Capacity}

When the receiver has complete knowledge of the channel and the transmitter does not, the channel capacity can be determined by the celebrated Foschini-Telatar equation [2]:

$$
C=\log _{2}\left[\operatorname{det}\left(I+\frac{\rho}{n} \mathbf{R}\right)\right]=\sum_{i=1}^{n} \log \left(1+\frac{\rho}{n} \lambda_{i}\right) \mathrm{bit} / \mathrm{s} / \mathrm{Hz}
$$

where $\rho$ is the average SNR at the receiver, and $\lambda_{i}$ is the ith eigenvalue of the correlation matrix. 


\section{B. Diversity Gain}

The diversity gain model assumes the use of maximal ratio combining (MRC) at the receiver [3]. The transmitter and receiver contain one and $n$ antenna elements respectively. The equation for the channel gains is the same as (1) with $j$ set to 1 . After generating enough samples of the channel, the outage probability at a prescribed SNR $\gamma_{p}$ can be determined as:

$$
P_{o}=\operatorname{Pr}\left(\gamma<\gamma_{p}\right)
$$

where $\operatorname{Pr}$ denotes the probability, $\gamma$ is the instantaneous SNR. The diversity gain can be obtained as:

$$
g=\gamma_{p d}\left(P_{o}\right)-\gamma_{p 1}\left(P_{o}\right)
$$

where $\gamma_{p d}\left(P_{0}\right)$ and $\gamma_{p 1}\left(P_{0}\right)$ are the SNR values for a prescribed outage probability for the diversity combining system and one-antenna system respectively.

\section{AOA Distributions}

Multipath signals arrive at the receiver at angles that are described in the model by AOA distributions. Two categories of AOA distributions are investigated continuous and impulsive ones. The continuous distributions include truncated versions of the uniform, Gaussian, Laplacian, and cosine distributions, as well as the circular scatterer model. The impulsive models describe distributions where the AOAs are limited to a few fixed directions. The AOA in both categories are limited to $\pm 90^{\circ}$ of the mean AOA, which is assumed to be the same as the broadside direction. Figure 1 shows all the continuous AOA distributions studied in this paper.

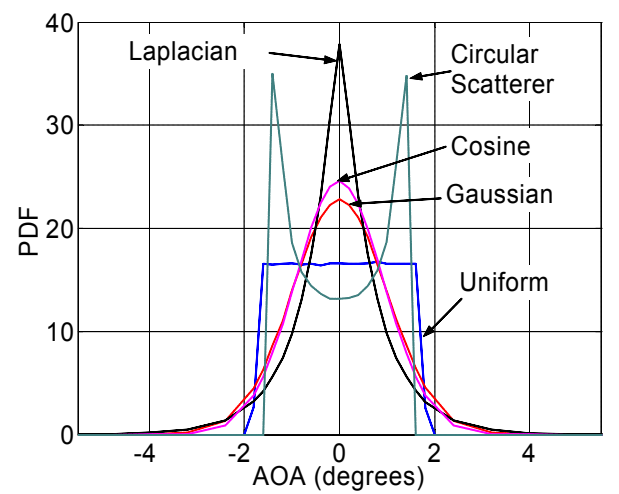

Figure 1. AOA Distributions (angular spread $=1$ degree)

Some of the AOA distributions discussed in this paper have been used before [4-8]. However, models presented by different papers are often different, which complicates the efforts to compare the results and judge their generality. A major focus of this paper is to present comparative results for all discussed AOA distributions based on the same assumptions and geometric references. This gives new insights, which were not available before.

\section{1) Truncated Uniform Distribution}

The PDF of the truncated uniform distribution is

$$
p(\varphi)=\frac{1}{2 \Delta} \text { for }-\Delta+\varphi_{0} \leq \varphi \leq \Delta+\varphi_{0}, 0 \text { elsewhere }
$$

where $\Delta$ is the one-sided range of the distribution. Angular spread of the truncated uniform distribution, defined as the standard deviation, is $S_{\varphi}=\Delta / \sqrt{3}$.

\section{2) Truncated Gaussian Distribution}

The PDF of the truncated Gaussian distribution is

$$
p(\varphi)=\left(Q / \sqrt{2 \pi \sigma^{2}}\right) e^{\frac{\left(\varphi-\varphi_{0}\right)^{2}}{2 \sigma^{2}}},-\frac{\pi}{2}+\varphi_{0} \leq \varphi \leq \frac{\pi}{2}+\varphi_{0},(7)
$$

where

$$
Q=1 / \operatorname{erf}(\pi / \sqrt{8} \sigma)
$$

and

$\operatorname{erf}(x)=(2 / \sqrt{\pi}) \int_{0}^{x} \exp \left(-t^{2}\right) d t$. Angular spread can be shown to be:

$$
S_{\varphi}=\left\{Q \sigma^{2}\left[-\sqrt{\frac{\pi}{2}} \frac{1}{\sigma} \exp \left(\frac{\pi^{2}}{8 \sigma^{2}}\right)+\operatorname{erf}\left(\frac{\pi}{\sqrt{8} \sigma}\right)\right]\right\}^{1 / 2}
$$

\section{3) Truncated Laplacian Distribution}

The PDF of the truncated Laplacian distribution is

$$
p(\varphi)=\left(Q / \sqrt{2 \pi \sigma^{2}}\right) e^{\frac{\sqrt{2}\left|\varphi-\varphi_{0}\right|}{\sigma}},-\frac{\pi}{2}+\varphi_{0} \leq \varphi \leq \frac{\pi}{2}+\varphi_{0}
$$

where $Q=(1-\exp (-\pi / \sqrt{2} \sigma))^{-1}$. Angular spread can be shown to be:

$$
S_{\varphi}=\left\{Q\left[-e^{\pi / \sqrt{2} \sigma}\left(\frac{\pi^{2}}{4}+\frac{\pi \sigma}{\sqrt{2}}+\sigma^{2}\right)+\sigma^{2}\right]\right\}^{\frac{1}{2}}
$$

\section{4) Truncated Cosine Distribution}

The PDF of truncated Cosine distribution is:

$$
p(\varphi)=Q \cos ^{n}\left(\varphi_{0}\right) \text { for }-\frac{\pi}{2}+\varphi_{0} \leq \varphi \leq \frac{\pi}{2}+\varphi_{0}
$$

where for even and odd $\mathrm{n}$ respectively,

$$
Q_{\text {even }}=\frac{\pi}{2^{2 n}}\left(\begin{array}{c}
2 n \\
n
\end{array}\right), Q_{\text {odd }}=\sum_{k=0}^{n}\left(\begin{array}{c}
2 n+1 \\
k
\end{array}\right) \frac{2(-1)^{n-k}}{2 n-2 k+1}
$$

Angular spread for even and odd $\mathrm{n}$ can be shown to be:

$$
\begin{aligned}
S_{\varphi, e v} & =\left[\frac{2}{3 \pi}\left(\frac{\pi}{2}\right)^{3}+8\left(\begin{array}{c}
2 n \\
n
\end{array}\right)^{-1} \sum_{k=0}^{n-1}\left(\begin{array}{c}
2 n \\
k
\end{array}\right) \frac{(n-k)(-1)^{n-k}}{(2 n-2 k)^{3}}\right]^{\frac{1}{2}} \\
S_{\varphi, \text { odd }}=\left[A^{-1} Q_{\text {odd }}\right]^{-1 / 2} & \\
A & =\sum_{k=0}^{n}\left(\begin{array}{c}
2 n+1 \\
k
\end{array}\right)^{2(-1)^{n-k}\left([(2 n-2 k+1) \pi / 2]^{2}-2\right)}
\end{aligned}
$$




\section{5) Circular Scatterer Model}

The circular scatterer model [7] emulates a practical wireless environment, where the transmitted signals bounce off a circular ring of equivalent scatterers surrounding the transmitter, and head towards the receiver. The AOA is given by

$$
\varphi(L)=\sin ^{-1}(R \sin \alpha / L)
$$

where $\mathrm{L}$ is the distance between scatterer and the Rx. The PDF of circular scatterer model is:

$$
p(\varphi) \approx \frac{D}{\pi R}\left(1-\frac{D^{2} \varphi^{2}}{R^{2}}\right)^{-1 / 2}, \varphi \in\left[-\varphi_{\max }, \varphi_{\max }\right]
$$

where $\mathrm{D}$ is the distance between the receiver and the transmitter (i.e. center of the circle of scatterer), and $\mathrm{R}$ is the radius of the circle of scatterers. (note that there are 2 different scatterers corresponding to the same $\varphi$; for more details, see Fig. 2 in [7]). Eq. (15) is valid for most practical situations when the radius of circle is much smaller than the distance between transmitter and receiver, $R / D<<1$. The angular spread under this condition can be approximated by $\sigma_{\varphi}=\Delta / \sqrt{2}$ where $\Delta$ is the maximum AOA.

\section{6) Impulsive Models}

Impulsive models emulate wireless environments where the multipath signals arrive exclusively from a limited number of fixed directions. Traditional studies often assume a rich scattering environment with large numbers of multipath signals arriving at the receiver. It would be of interest to find out the effects on MIMO systems if this condition does not hold. The PDF of this model for odd and even $\mathrm{N}$ is:

$$
\begin{gathered}
p_{\text {odd }}(\varphi)=\frac{1}{N}\left[\sum_{n=1}^{(N-1) / 2}\left[\delta\left(\varphi-\frac{2 n \Delta}{N-1}\right)+\delta\left(\varphi+\frac{2 n \Delta}{N-1}\right)\right]+\delta(\varphi)\right] \\
p_{\text {even }}(\varphi)=\frac{1}{N} \sum_{n=1}^{N / 2}\left[\delta\left(\varphi-\frac{2 n \Delta}{N}\right)+\delta\left(\varphi+\frac{2 n \Delta}{N}\right)\right]
\end{gathered}
$$

where $\mathrm{N}$ is the number of unique AOA in the AOA distribution, $\delta(\varphi)$ is the delta function, and $\Delta$ is the onesided range of the distribution.

\section{CORRELATION, CAPACITY AND DIVERSITY GAIN: RESULTS}

\section{A. Continuous Distributions}

Larger separation between receive antenna elements results in better capacity performance. This trend is observed in the capacity curves of the continuous distributions. Capacity increases up to the maximum determined by the number of antenna elements and the SNR. After reaching the maximum value, the capacity for most AOA distributions stays constant, while some others exhibit minor fluctuations around the maximum. In the rest of this paper, the transient region refers to the range of antenna spacing at which the capacity is rising and has not yet reached $90 \%$ of the maximum; the saturation region refers to the range of antenna spacing at which the capacity has reached $90 \%$ of the maximum.

Figure 2 shows the capacity curves for all five continuous distributions. The AOA distributions with a large concentration of signals in the mean AOA (Gaussian, Laplacian, and Cosine) exhibit almost identical trend in capacity. They exhibit the fastest rising slopes in the transient region and reaches saturation at the lowest values of antenna spacing. All three distributions do not exhibit fluctuations in the saturation region. For the uniform AOA distribution, the capacity enters saturation region at a slightly bigger value of antenna spacing. The increase in capacity is also at a slower rate in the transient region. There are some minor fluctuations around the maximum capacity at the early stage of the saturation region. The AOA distribution with concentrations of signals at the edges (circular scatterer) exhibits the worst performance. It reaches the maximum at a large value of antenna spacing, and shows substantial amounts of fluctuations in the saturation region before finally converging to the maximum value.

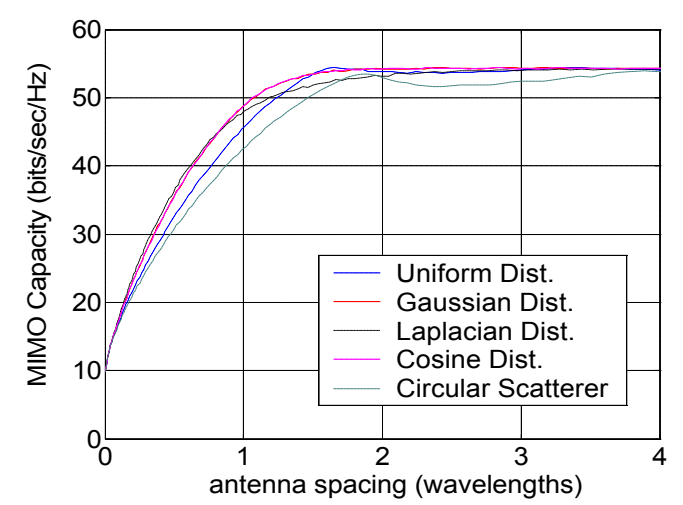

Figure 2. MIMO Capacity vs. Antenna Spacing

For the sake of fair comparison between different distributions, their parameters were chosen in such a way that the angular spread is the same for all the distributions. Simulations were performed on all five continuous distributions and various values of the angular spread. It is observed that when large values of angular spread are used, all the distributions reach the maximum capacity at smaller values of antenna spacing. However, the trend of the capacity curve of each AOA distribution relative to other AOA distributions remains the same.

More insight into the relationship between AOA distribution shape and capacity can be obtained by studying the correlation of the signals received by adjacent antenna elements. Figure 3 shows the correlation curves of all five continuous distributions studied in this paper. It is clear that the trends observed in the capacity curves are reflected in the correlation curves. The antenna spacing at which a particular distribution reaches the lowest correlation is the 
same antenna spacing at which it first reaches the maximum capacity. The distributions with fluctuations about zero correlation are the same distributions that produce fluctuations around the maximum capacity. There exists an explicit relationship between correlation and capacity in MIMO systems, even for rather complicated scattering environments.

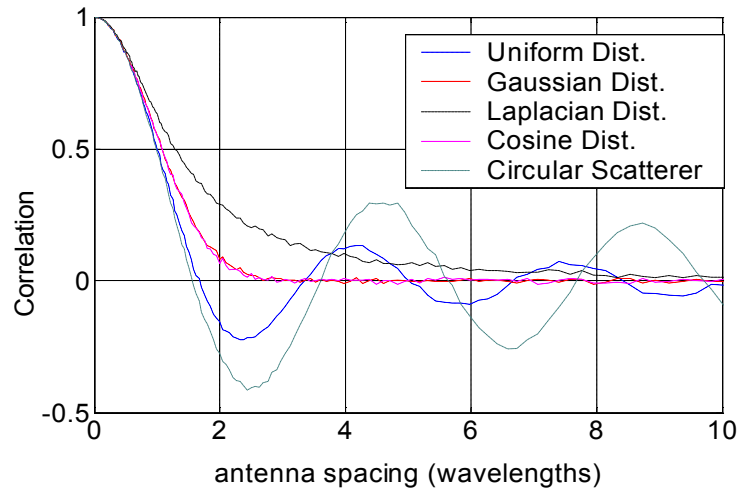

Figure 3. Correlation vs. Antenna Spacing

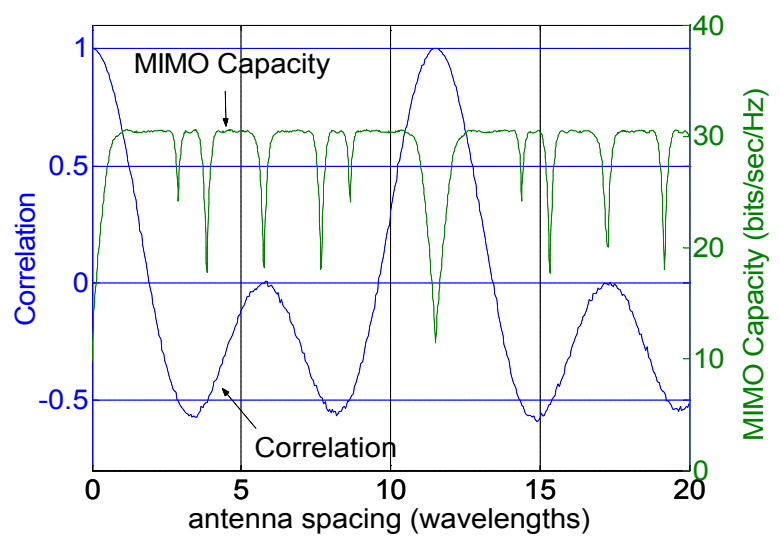

Fig. 4 Correlation and Capacity for 4-path impulsive AOA

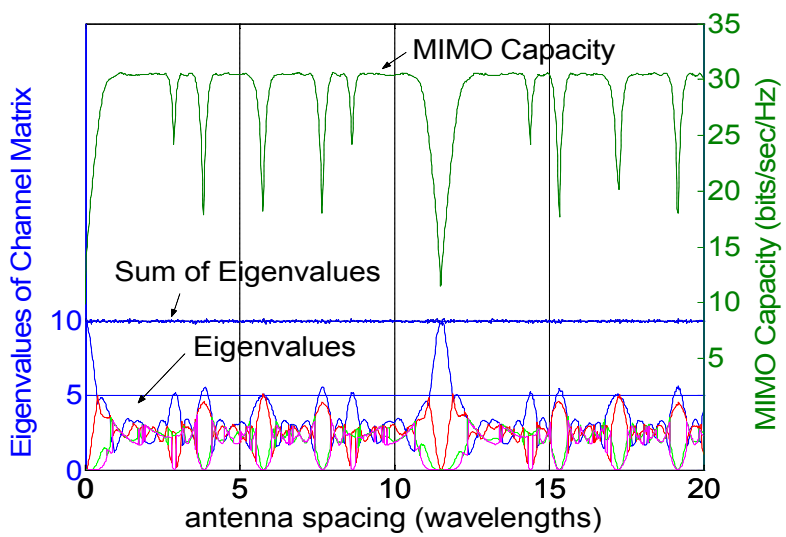

Fig. 5. Eigenvalues and Capacity for 4-path impulsive AOA
The angular spread of an AOA distribution and mean AOA are also studied in the simulations. It is observed that for any given AOA distribution, as angular spread increases, the slope of the capacity curve increases, and maximum capacity will be reached at a smaller value of antenna spacing. Similar trends are observed as the mean AOA moves away from the broadside direction.

\section{B. Impulsive Models}

The capacity curves obtained using the impulsive models are quite different. The curves contain periodical oscillations as antenna spacing increases. Also, as the number of unique AOA increases, a higher value of maximum capacity is attained. The lowest value of the drops in the capacity curve is always equal to the highest achievable capacity of another distribution with less AOAs. It points to the fact that there exist distinct levels of capacity when the multipath signals are limited to a few unique directions.

The correlation functions of impulsive models are periodic functions and do not saturate to zero at large values of antenna spacing. For illustration purpose, the correlation function of the two-path impulsive model is:

$$
r=\cos (2 \pi d \sin (\Delta))
$$

where $\mathrm{d}$ is the antenna spacing, and $\Delta$ is the one-sided range of the AOA distribution. Its oscillating nature in $\mathrm{d}$ is clear. Fig. 4 shows the correlation and capacity of the impulsive AOA distribution of the 4-path impulsive AOA. The maxima and minima of the correlation coincide with some of the drops in the capacity, because they represent extreme values of correlation that would result in low capacity. The correlation studied here is between signals received by adjacent antenna elements, and the correlations between signals received by antenna elements that are more than one element apart are not taken into account here. This is the reason why some of the drops in capacity do not have a corresponding maxima or minima in correlation.

Fig. 5 shows the eigenvalues and capacity of the delta function AOA distribution with four unique AOAs. It is observed that the maximum number of non-zero eigenvalues is the same as the number of unique AOA. Each eigenvector of the channel matrix represents the subspace of an incoming signal that can be detected by the receiver. Thus the number of non-zero eigenvalues is the number of independent paths that can be received by the $\mathrm{Rx}$, with the maximum being equal to the number of unique AOAs. Even though the amplitudes of these eigenvalues vary as function of antenna spacing, the sum of the eigenvalues at any given antenna spacing is always equal to the total number of antenna elements in the receiver, due to the normalization adopted.

There is a clear relationship between the fluctuations in the capacity and the fluctuations in the eigenvalues. The highest capacity is attained when all the eigenvalues are non-zero. The lowest capacity is attained when there is only 
one non-zero eigenvalues. When the number of non-zero eigenvalues is less than the maximum value but larger than one, the capacity drops to the maximum value of capacity for systems with that number of non-zero eigenvalues. Thus, there exist distinct levels of capacity.

\section{Diversity Combining}

Simulations have shown that the AOA distribution shapes have negligible effects on the outage probability and diversity gains when maximal ratio combining is implemented in MIMO systems. Intuitively, this can be explained by the following facts: (i) as Fig. 3 demonstrates, the distribution shape significantly affects only low correlation (i.e. $|r| \leq 0.5$ ), (ii) low correlation does not have significant effect on the diversity gain/outage probability. Fig. 6 shows the outage probability vs. antenna spacing graph for different AOA distributions when the SNR is fixed to $10 \mathrm{~dB}$ below the average SNR. Clearly, the four distributions result in very similar outage probability curves. Since distribution shape has negligible impact on outage probability, the same conclusion applies to diversity gain.

Contrary to the distribution shape, the angular spread and the mean AOA have significant impact on the diversity gain. Changes in the angular spread and mean AOA affect the correlation [1], which in turn, affects diversity gain. Regardless of a particular scattering scenario, the diversity gain versus correlation can be approximated as

$$
D \approx D_{0}\left(1-|r|^{2}\right)
$$

where $\mathrm{r}$ is the normalized correlation coefficient, and $D_{0}$ is the maximum gain (when $r=0$, i.e. large spacing).

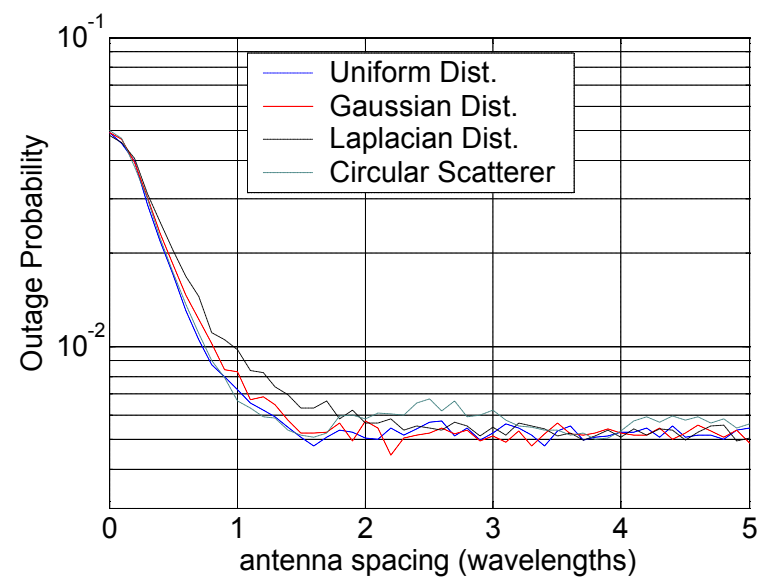

Fig. 6. Outage Probability vs. Antenna Spacing Graph

\section{CONCLUSION}

We presented an analysis of the impact of multipath angular distribution on the channel correlation and the performance of MIMO systems. Two classes of AOA distributions are studied in this paper: continuous and impulsive. It was demonstrated that they have dramatically different effect on the correlation (and, hence, capacity and diversity gain): while continuous distributions result in correlation decreasing with antenna spacing and finally approaching zero for large spacing, the impulsive distributions results in periodic oscillations of the correlation, with the peaks being far away from zero for arbitrary large spacing. Impulsive models limit the AOAs to a few directions, thus reducing the number of degrees of freedom of the channel (i.e. nonzero eigenvalues) and placing a limit on the capacity.

The angular distribution shape has a noticeable but minor effect on the correlation, capacity and diversity gain. The major effect is due to the angular spread. Intuitively, this can be explained by the fact that the correlation is an integral of the angular PDF. Hence, all the local features are smoothed out.

For a given angular spread, distributions with concentrations of signals at the mean AOA tend to achieve the maximum capacity at lower values of antenna spacing than the other distributions. They do not exhibit fluctuations which are present in the other distributions.

After the studies were finished and the paper has been prepared, we learned that somewhat similar studies have been reported in [9].

\section{REFERENCES}

[1] S. Loyka, G. Tsoulos, "Estimating MIMO System Performance Using the Correlation Matrix Approach", IEEE Communications Letters, v.6, N. 1, January 2002.

[2] G.J. Foschini and M.J. Gans, "On Limits of Wireless Communications in a Fading Environment when Using Multiple Antennas", Wireless Personal Communications, v.6, N. 3, March 1998.

[3] W.C. Jakes Jr., Microwave Mobile Communication. New York: Wiley, 1974

[4] J.Fuhl, A.F. Molisch and E.Bonek, "Unified channel model for mobile radio systems with smart antennas", IEE Proc Rad., Sonar, Nav., v.145, N.1, Feb 1998

[5] L. Schumacher et al, "From Antenna Spacings to Theoretical Capabilities - Guidelines for simulating MIMO Systems", IEEE International Symposium on Personal, Indoor and Mobile Radio Comm, v.2, Sept. 2002

[6] K. I. Pedersen et al, "Spatial Channel Characteristics in Outdoor Environments and their Impact on BS Antenna System Performance", IEEE Vehicular Technology Conference 1998, v.2, May 1998

[7] R.B. Ertel, J.H. Reed, "Angle and time of arrival statistic for circular and elliptical scattering models", IEEE Journal on Selected Areas in Communications, v. 17, N. 11, Nov. 1999

[8] W.C.Y. Lee, 'Effects on correlation between two mobile radio base-station antennas', IEEE Transactions on Communications, 1973

[9] R. M. Buehrer, The Impact of Angular Energy Distribution on Spatial Correlation, IEEE Vehicular Technology Conference, Vancouver, Canada, September 24-28, 2002, pp.1173-1177. 